\title{
Noise-induced transitions vs. noise-induced phase transitions
}

\author{
Raul Toral \\ IFISC (Instituto de Física Interdisciplinar y Sistemas Complejos), CSIC-UIB, E-07122 Palma de \\ Mallorca, Spain
}

\begin{abstract}
I will briefly review the field of noise-induced phase transitions, emphasizing the main differences with the phase-induced transitions and showing that they appear in different systems. I will show that a noise-induced transition can disappear after a suitable change of variables and I will also discuss the breaking of ergodicity and symmetry breaking that occur in noise-induced phase transitions in the thermodynamic limit, but not in noise-induced transitions.
\end{abstract}

Keywords: Non-equilibrium transitions, fluctuations, finite-size effects.

PACS: 05.40.-a,74.40.Gh,05.10.Gg,64.60.Cn

\section{BIFURCATIONS IN STOCHASTIC SYSTEMS}

A bifurcation in a dynamical system is a change in the number of fixed points, or in their relative stability, that occurs when varying a control parameter, the so-called bifurcation parameter. The value of this parameter at which the change occurs is the bifurcation point [1]. The normal form of a bifurcation is the simplest mathematical model (usually involving polynomials of the lowest possible order) for which a particular change of behavior occurs. One of the simplest examples is that of the transcritical bifurcation for which the normal form is $d x(t) / d t=\mu x-x^{2}$, the Verhulst, or logistic, equation [2]. This equation can model, for instance, the growth of biological populations, or autocatalytic reactions, amongst other applications. For $\mu<0$, there is only one (stable) fixed point at $x=0$, whereas for $\mu>0$ there are two fixed points: the one at $x=0$ (which is now unstable) and another one at $x=\mu$ which is stable. Another simple example is that of the supercritical pitchfork bifurcation for which the normal form is $d x(t) / d t=\mu x-x^{3}$, the Landau equation used in the context of phase transitions in the mean-field approach. For $\mu<0$, there is only one (stable) fixed point at $x=0$, whereas for $\mu>0$ there are three fixed points: the one at $x=0$ (which is now unstable) and two more at $x= \pm \sqrt{\mu}$ which are stable. In both examples, the bifurcation point is, hence, $\mu=0$. The importance of the stable fixed points is that, under some additional conditions, they determine the long-time dynamical behavior, as the dynamical evolution tends to one of the stable fixed points, and then it stops [3]. In the supercritical pitchfork, the value $x=+\sqrt{\mu}$ is reached if the initial condition is $x(t=0)>0$, whereas the fixed point at $x=-\sqrt{\mu}$ is reached whenever $x(t=0)<0$. The symmetry $x \rightarrow-x$ of the differential equation is broken by the initial condition in the case $\mu>0$.

When there are stochastic, so-called noise, terms in the dynamics, usually there are no fixed points but the long-time dynamical behavior still has some preferred values. Consider, for example, the normal form for the supercritical bifurcation with an additional 
noise term

$$
\frac{d x(t)}{d t}=\mu x-x^{3}+\sqrt{2 D} \xi(t),
$$

being $\xi(t)$ a Gaussian process of zero mean and correlations $\left\langle\xi(t) \xi\left(t^{\prime}\right)\right\rangle=\delta\left(t-t^{\prime}\right)$, or white noise [3]. $D$ is the noise intensity. This equation can be written in terms of relaxational dynamics [3] in a double-well potential $V(x)$ :

$$
\frac{d x(t)}{d t}=-\frac{\partial V(x)}{\partial x}+\sqrt{2 D} \xi(t), \quad V(x)=-\frac{\mu}{2} x^{2}+\frac{1}{4} x^{4} .
$$

It is possible to prove using the Fokker-Planck equation [4] (see later) that the stationary probability distribution for the $x$ variable is $P_{\mathrm{st}}(x)=\mathscr{Z}^{-1} \exp \left[-\frac{V(x)}{D}\right]$, being $\mathscr{Z}=$ $\int_{-\infty}^{\infty} d x \exp \left[-\frac{V(x)}{D}\right]$ a normalization factor. The stationary probability has maxima at $x=0$ for $\mu<0$ and at $x= \pm \sqrt{\mu}$ for $\mu>0$. So it is still true that, from a probabilistic point of view, the fixed points of the deterministic, i.e. $D=0$, dynamics are the ones preferred by the stochastic trajectories, but the dynamics does not end in one of the fixed points. Another important difference with the deterministic dynamics is that, for $\mu>0$, the trajectories are not confined to the neighborhood of one of the maxima. There are constant jumps between the two maxima of the probability distribution. A classical calculation by Kramers [5], shows that the frequency of the jumps between the two maxima is proportional to $\exp \left[-\frac{\Delta V}{D}\right]$, being $\Delta V$ the height of the potential barrier between the maxima, or $\Delta V=\mu^{2} / 4$ in the double well potential. As there are many jumps between the maxima, the noise terms have restored the symmetry $x \rightarrow-x$ of the equation.

There are other more complicated examples. Consider, for example, the Verhulst equation with the addition of a noise term $\xi$ which is coupled multiplicatively to the dynamical variable $x$ :

$$
\frac{d x(t)}{d t}=\mu x-x^{2}+\sqrt{2 D} x \xi(t) .
$$

This can be thought as originated from the fact that the parameter $\mu$ randomly fluctuates and can be replaced by $\mu \rightarrow \mu+\sqrt{2 D} \xi(t)$. There are some mathematical subtleties to handle the presence of the singular function $\xi(t)$. After all, the correlation function of $\xi(t)$ is a delta function, a not too well defined mathematical object. The different possible interpretations of the integral $\int d t g(x(t)) \xi(t)$, for an arbitrary function $g(x)$, lead to different results. We will limit our considerations to the so-called Stratonovich interpretation $[6,7]$. In this example, $x=0$ is a fixed point of the stochastic dynamics. Therefore starting from $x(t=0)>0$ as it is the case in the biological or chemical applications, the barrier $x=0$ can never be crossed. For $\mu<0$, the value $x=0$ is an attracting boundary [6]: it will be reached in the asymptotic limit $t \rightarrow \infty$. As a consequence, the stationary probability distribution is $P_{\mathrm{st}}(x)=\delta(x)$. As $\mu$ increases and crosses zero, the picture changes. The full analysis uses the Fokker-Planck equation for the time dependent probability density $P(x, t)$. The stationary distribution for $0<\mu<D$ is no longer a delta function at $x=0$ but still has a maximum at $x=0$. However, when $\mu>D$, the maximum of $P_{\mathrm{st}}(x)$ is no longer at $x=0$ but it moves to $x=\mu-D$. 
Alternatively, for fixed $\mu>0$ one finds that the maximum of the stationary distribution switches from $x=\mu-D$ for $0<D<\mu$ to $x=0$ for $D>\mu$. Note that this is a somewhat counterintuitive result in the sense that a large value of the noise intensity leads to a state where the maximum of the distribution is located at a state, $x=0$, in which the noise term $x \xi(t)$ vanishes.

Similar shifts of the maxima of the probability distribution as the noise intensity increases appear in a large class of stochastic differential equations. They have been named generically as noise-induced transitions [8]. In the general case of a stochastic differential equation of the form $d x(t) / d t=q(x)+\sqrt{2 D} g(x) \xi(t)$, the Fokker-Planck equation reads:

$$
\frac{\partial P(x, t)}{\partial t}=-\frac{\partial}{\partial x}\left[\left(q(x)-D g(x) g^{\prime}(x)\right) P(x, t)\right]+D \frac{\partial^{2}}{\partial x^{2}}\left[g(x)^{2} P(x, t)\right]
$$

and the steady-state solution $\left.\frac{\partial P(x, t)}{\partial t}\right|_{P=P_{\mathrm{st}}}=0$ is:

$$
P_{\text {st }}(x)=\mathscr{Z}^{-1} \exp \left[\int^{x} d x^{\prime} \frac{q\left(x^{\prime}\right)-D g\left(x^{\prime}\right) g^{\prime}\left(x^{\prime}\right)}{D g^{2}\left(x^{\prime}\right)}\right] .
$$

The maxima $\bar{x}$ of this distribution are given by

$$
q(\bar{x})-D g(\bar{x}) g^{\prime}(\bar{x})=0 .
$$

And it is clear that $\bar{x}(D)$ depends on the noise intensity $D$. There are examples [8] in which equations that display the $x \rightarrow-x$ symmetry are such that for small noise intensity $D$ the distribution is unimodal centered at $\bar{x}=0$, and that increasing $D$ the distribution becomes bimodal with maxima at $\pm \bar{x}(D) \neq 0$. This is the generic behavior whenever $q(x)=-x+o(x)$ and $g(x)=1+x^{2}+o\left(x^{2}\right)$. A specific example is Hongler's model [9] $q(x)=-\tanh (x), g(x)=\operatorname{sech}(x)$. The transition occurs at $D=D_{c}=1$. The situation, in principle, could be considered the equivalent of the supercritical pitchfork bifurcation, in the sense that the most visited states are $x=0$ for $D<1$ and $\pm \bar{x}(D)$ for $D>1$. However, the same remarks than in the case of the model of Eq.(1) apply: the bifurcation does not break the $x \rightarrow-x$ symmetry, as trajectories visit ergodically all possible values of $x$ and, therefore, there are many jumps between the two preferred states. Furthermore, it is possible to show that the change in the number of maxima in the probability distribution is simply a matter of the variable used and that a simple change of variables can eliminate the bifurcation. This is explained in the next section.

\section{NOISE-INDUCED TRANSITIONS AS A CHANGE OF VARIABLES}

Let us consider the Gaussian distribution:

$$
f_{z}(z)=\frac{1}{\sqrt{2 D \pi}} e^{-z^{2} / 2 D} .
$$

It is obviously single-peaked for all values of $D$, the noise intensity. Let us now introduce the new variable $x=\operatorname{argsh}(z)$ or $z=\sinh (x)$. The change of variables (i) does not depend 
on the noise intensity $D$ and (ii) it is one-to-one, mapping the set of real numbers onto itself. The probability distribution for the new variable is

$$
f_{x}(x)=f_{z}(z)\left|\frac{d z}{d x}\right|=f_{z}(z) \cosh (x)
$$

or

$$
f_{x}(x)=\frac{1}{\sqrt{2 D \pi}} e^{-\left[\sinh (x)^{2}-2 D \ln \cosh (x)\right] / 2 D} \equiv \frac{1}{\sqrt{2 D \pi}} e^{-\frac{V_{\text {eff }}(x)}{D}},
$$

with an effective potential

$$
V_{\mathrm{eff}}(x)=\frac{1}{2} \sinh (x)^{2}-D \ln \cosh (x)
$$

which depends on the noise intensity. The potential is monostable for $D<D_{c}$ and bistable for $D>D_{c}$ with $D_{c}=1$, as the expansion $V_{\mathrm{eff}}(x)=\frac{1-D}{2} x^{2}+\frac{2+D}{12} x^{4}+O\left(x^{6}\right)$ shows. The Horsthemke-Lefever mechanism for noise-induced transitions is an equivalent way of reproducing this result. Just take the Langevin equation:

$$
\frac{d z}{d t}=-z+\sqrt{2 D} \xi(t)
$$

being $\xi(t)$ zero-mean white noise, $\left\langle\xi(t) \xi\left(t^{\prime}\right)\right\rangle=\delta\left(t-t^{\prime}\right)$. Its steady-state probability is

$$
f_{z}(z)=\mathscr{Z}^{-1} e^{-\frac{V(z)}{D}}
$$

with a potential function $V(z)=\frac{z^{2}}{2}, \mathscr{Z}$ is a normalization constant.

We now perform the aforementioned change of variables $x=\operatorname{argsh}(z)$ to obtain (Stratonovich sense)

$$
\frac{d x}{d t}=-\tanh (x)+\operatorname{sech}(x) \sqrt{2 D} \xi(t)
$$

which is Hongler's model, one of the typical examples of noise-induced transitions explained above.

This result is very general. The same (well-known) trick can be used to reduce any one-variable Langevin equation with multiplicative noise:

$$
\frac{d x}{d t}=q(x)+g(x) \sqrt{2 D} \xi(t)
$$

to one with additive noise. Simply make the change of variables defined by $d z=d x / g(x)$ or $z=\int^{x} d x^{\prime} / g\left(x^{\prime}\right)$ to obtain

$$
\frac{d z}{d t}=F(z)+\sqrt{2 D} \xi(t)
$$

with

$$
F(z)=q(x) / g(x),
$$


expressed in terms of the variable $z$. The steady-state distribution of $z$ can be written as

$$
f_{z}(z)=\mathscr{Z}^{-1} e^{-\frac{V(z)}{D}}
$$

with a potential

$$
V(z)=-\int^{z} d z^{\prime} F\left(z^{\prime}\right)
$$

The steady-state probability distribution in terms of the variable $x$ (assuming a one-toone change of variables) is

$f_{x}(x)=f_{z}(z)\left|\frac{d z}{d x}\right|=\frac{f_{z}(z)}{|g(x)|}=\frac{\mathscr{Z}-1}{|g(x)|} e^{\frac{1}{D} \int^{z} d z^{\prime} F\left(z^{\prime}\right)}=\frac{\mathscr{Z}^{-1}}{|g(x)|} e^{\frac{1}{D} \int^{x} \frac{d x^{\prime}}{g\left(x^{\prime}\right)} \frac{q\left(x^{\prime}\right)}{g\left(x^{\prime}\right)}}=\frac{\mathscr{Z}^{-1}}{|g(x)|} e^{\frac{1}{D} \int^{x} d x^{\prime} \frac{q\left(x^{\prime}\right)}{g\left(x^{\prime}\right)^{2}}}$,

the same steady-state probability distribution coming from the multiplicative-noise Langevin equation (14) that was written in Eq.(5). In terms of an effective potential: $f_{x}(x)=\mathscr{Z}^{-1} e^{-\frac{V \text { eff }(x)}{D}}$ we have

$$
V_{\mathrm{eff}}(x)=-\int^{x} d x^{\prime} \frac{q\left(x^{\prime}\right)}{g\left(x^{\prime}\right)^{2}}+D \ln |g(x)|
$$

A noise-induced transition will appear if the potential $V_{\text {eff }}(x)$ changes from monostable to bistable as the noise intensity $D$ increases.

Another widely used example of a noise-induced transition [8] is that of $q(x)=$ $-x+\lambda x\left(1-x^{2}\right)$ and $g(x)=1-x^{2}$. The change of variables $z=\int^{x} \frac{d x^{\prime}}{1-x^{\prime 2}}=\frac{1}{2} \log \left(\frac{1+x}{1-x}\right)$, or $x=\tanh (z)$ leads to the Langevin equation:

$$
\frac{d z}{d t}=-\sinh (z) \cosh (z)+\lambda \tanh (z)+\sqrt{2 D} \xi(t) .
$$

Note that $x \in(-1,1)$, a fact already implied in the original Langevin equation since $x= \pm 1$ are reflecting barriers. The steady-state probability distribution of this Langevin equation is $f_{z}(z)=\mathscr{Z}^{-1} e^{-\frac{V(z)}{D}}$ with a potential $V(z)=\frac{1}{2} \cosh (z)^{2}-\lambda \log (\cosh (z))$. The Taylor expansion $V(z)=\frac{1}{2}+\frac{1-\lambda}{2} z^{2}+\frac{2+\lambda}{12} z^{4}+O\left(z^{6}\right)$, shows that $f_{z}(z)$ has a single minimum at $z=0$ for $\lambda<1$ and double minima for $\lambda>1$. As far as the $x$ variable is concerned, the effective potential as given by (20) is

$$
V_{\mathrm{eff}}(x)=\frac{1}{2\left(1-x^{2}\right)}+\frac{\lambda+2 D}{2} \log \left(1-x^{2}\right)
$$

The Taylor expansion $V_{\text {eff }}(x)=\frac{1}{2}+\frac{1-\lambda-2 D}{2} x^{2}+\frac{2-\lambda-2 D}{4} x^{4}+O\left[x^{6}\right]$ shows that the potential leads to a monostable distribution if $\lambda+2 D<1$ and to a bistable one if $\lambda+2 D>1$. Hence, a noise-induced transition occurs for $\lambda<1$ since a bistable distribution for the $x$ variable appears for $D>D_{c}=(1-\lambda) / 2$. Note, however, that the distribution of the $z$ variable is monostable for all values of $D$, so that the noise-induced transition is dependent on the variable considered. In the case $\lambda>1$ the distribution is always bistable, both for the $x$ and the $z$ variables. 
The change $x=\tanh (z)$ also induces a transition in the simpler case that the $z$ variable follows the Gaussian distribution Eq.(7). The probability distribution function for the new variable is $q(x)=\frac{1+x^{2}}{\sqrt{2 D \pi}} e^{-\operatorname{argth}(x)^{2} / 2 D}=\frac{1}{\sqrt{2 D \pi}}\left[1+\left(1-\frac{1}{2 D}\right) x^{2}+O\left(x^{4}\right)\right]$ which indicates a phase transition at $D_{c}=1 / 2$.

A remarkable example is the change $x=\frac{z}{1+|z|}$ which leads to a probability distribution $q(x)=\frac{1}{\sqrt{2 D \pi}} \frac{e^{-\left(\frac{x}{1-|x|}\right)^{2} / 2 D}}{(1-|x|)^{2}}$ for $x \in(-1,1)$ which is bimodal for any $D>0$, or $D_{c}=0$.

\section{NOISE-INDUCED PHASE TRANSITIONS}

How can one obtain a true, symmetry breaking, bifurcation in a stochastic model? The answer lies in the coupling of many individual systems in order to obtain a bifurcation in the macroscopic variable. Let us explain this with a simple example: the standard Ginzburg-Landau model for phase transitions [10]. It consists of many coupled dynamical variables $x_{i}(t), i=1, \ldots, N$ which individually follow Eq.(1). The full model is:

$$
\frac{d x_{i}(t)}{d t}=\mu x_{i}-x_{i}^{3}+\frac{C}{N_{i}} \sum_{j \in \mathscr{N}_{i}}\left(x_{j}-x_{i}\right)+\sqrt{2 D} \xi_{i}(t) .
$$

The noise variables are now independent Gaussian variables of zero mean and correlations $\left\langle\xi_{i}(t) \xi_{j}\left(t^{\prime}\right)\right\rangle=\delta_{i j} \delta\left(t-t^{\prime}\right) . \mathscr{N}_{i}$ refers to the set of $N_{i}$ variables $x_{j}$ which are coupled to $x_{i}$. Typical situations include an all-to-all coupling where $\mathscr{N}_{i}$ is the set of all units and $N_{i}=N$, or regular $d$-dimensional lattices where a unit $x_{i}$ is connected to the set of $N_{i}=2 d$ nearest neighbors, although in more recent applications one also considers nonregular, random, small world, scale free or other types of lattices [11]. $C$ is the coupling constant. If $C=0$ each unit is independent of the other and displays the stochastic bifurcation at $\mu=0$ explained before. For $C>0$, a collective state can develop in which the global variable $m(t)=N^{-1} \sum_{i=1}^{N} x_{i}(t)$ follows, in the thermodynamic limit, a true bifurcation from a state in which the stationary distribution is $P_{\mathrm{st}}(x)=\delta(m)$, to another one in which it is either $P_{\mathrm{st}}(x)=\delta\left(m-m_{0}\right)$ or $P_{\mathrm{st}}(x)=\delta\left(m+m_{0}\right)$. This is nothing but a phase transition. Here, borrowing the language from the para-ferromagnetic transition [12], $m_{0}$ is called, in this context, the spontaneous magnetization and it is a function of noise intensity $D$, coupling constant $C$ and the parameter $\mu$. It is important to stress that a true symmetry-breaking transition, with non-ergodic behavior, occurs only in the thermodynamic limit $N \rightarrow \infty$. For finite $N$ the stationary probability distribution $P_{\mathrm{st}}(m)$ is either a function peaked around $m=0$ or displays two large maxima around $\pm m_{0}$. The height of these maxima increases with $N$ and the width around them decreases with $N$ until delta-functions are reached for $N \rightarrow \infty$. One can see evidence of this behavior in Fig. 1 The price one has to pay to obtain this symmetry-breaking bifurcation is that, for fixed $C$ and $D$, the bifurcation point is no longer at $\mu=0$, but is is shifted to a positive value $\mu_{c}$ [13]. Alternatively, for fixed $\mu>0$ there is a bifurcation induced by varying the noise intensity: when $D<D_{c}$ (the critical noise intensity), the distribution of $m$ is a delta function located either at $m= \pm m_{0}$; for $D>D_{c}$, the distribution is again a delta function around $m=0$. The bifurcation acts in the way noise is expected to influence 
the dynamics: for larger noise intensity the distribution is peaked around $m=0$ (a situation in which roughly half of the $x_{i}$ variables have a positive value and the other half negative, or disordered). When the noise intensity is small, $D<D_{c}$, the distribution is peaked around $+m_{0}$ or $m_{0}$ and, hence, variables $x_{i}$ have a probability distribution peaked around this value, or ordered. As either $+m_{0}$ or $-m_{0}$ is selected (depending on initial conditions and realizations of the noise variables), the $x \rightarrow-x$ symmetry has been broken for $D<D_{c}$ and it is restored for $D>D_{c}$. It is not possible, in general, to obtain the probability distribution $p\left(x_{i}, t\right)$ for a single unit $x_{i}$, but an approximate result can be derived within the so-called Weiss effective-field theory $[14,12]$. In a nutshell, it consists in replacing the detailed interaction with the neighbors with the global variable $m(t)$. This leads to a single equation for $x_{i}$ :

$$
\frac{d x_{i}(t)}{d t}=\mu x_{i}-x_{i}^{3}+C\left(m(t)-x_{i}\right)+\sqrt{2 D} \xi_{i}(t) .
$$

From here it is possible to write the Fokker-Planck equation for $p\left(x_{i}, t\right)$. The stationary solution depends on the value of $m(t)$ in the steady state, $m_{0}$,

$$
p_{\mathrm{st}}\left(x_{i} ; m_{0}\right)=\mathscr{Z}^{-1} \exp \left[-v\left(x_{i} ; m_{0}\right) / D\right], \quad v(x ; m)=-C m_{0} x-\frac{\mu-C}{2} x^{2}+\frac{1}{4} x^{4}
$$

$m_{0}$ is obtained via the self-consistency relation $\langle x\rangle=\int d x p_{\mathrm{st}}\left(x ; m_{0}\right)=m_{0}$. This yields $m_{0}=m_{0}(D, C, \mu)$ and it is such that, for a range of values of $\mu$ and $\mu>C$, there is a critical value $D_{c}$ such that $m_{0}=0$ for $D>D_{c}$ and there are two solutions $\pm m_{0}$ with $m_{0}>0$ for $D<D_{c}$. Therefore, the one-unit dynamical system $x_{i}$ experiences a stochastic bifurcation, in the sense that the maxima of the probability of $p_{\mathrm{st}}\left(x_{i}\right)$ change location as $D$ crosses $D_{c}$.

The idea naturally arises of whether is is possible to obtain a bifurcation for the global variable if we couple $N$ units $\left(x_{1}, x_{2}, \ldots, x_{N}\right)$, each one of which experiences a noiseinduced transition from unimodal to bimodal as the noise intensity increases. In other words, if we consider the coupled system:

$$
\frac{d x_{i}(t)}{d t}=q\left(x_{i}\right)+\frac{C}{N_{i}} \sum_{j \in \mathscr{N}_{i}}\left(x_{j}-x_{i}\right)+\sqrt{2 D} g\left(x_{i}\right) \xi_{i}(t) .
$$

such that the uncoupled unit $\frac{d x_{i}(t)}{d t}=q\left(x_{i}\right)+\sqrt{2 D} g\left(x_{i}\right) \xi_{i}(t)$ undergoes a noise-induced transition in the sense of Hormthenske and Lefever, will the global variable $m(t)$ undergo a bifurcation from disorder to order as the noise intensity increases? The answer turns out to be no[15, 16], one of the reasons being that, as we have already noted, the shift in the maxima of the probability distribution of $p_{\text {st }}\left(x_{i}\right)$ might disappear after a change of variables, whereas a true bifurcation remains after a one-to-one change of variables.

However, it was found quite surprisingly $[15,16]$ that it is possible to find functions $q(x)$ and $g(x)$ such that the global variable $m(t)$ experiences a bifurcation from $m_{0}=0$ to $\pm m_{0}$ with $m_{0}>0$ increasing the noise intensity $D$. The minimal model (normal form) is

$$
\frac{d x_{i}(t)}{d t}=-x_{i}\left(1+x_{i}^{2}\right)^{2}+\frac{C}{N_{i}} \sum_{j \in \mathscr{N}_{i}}\left(x_{j}-x_{i}\right)+\sqrt{2 D}\left(1+x_{i}^{2}\right) \xi_{i}(t) .
$$



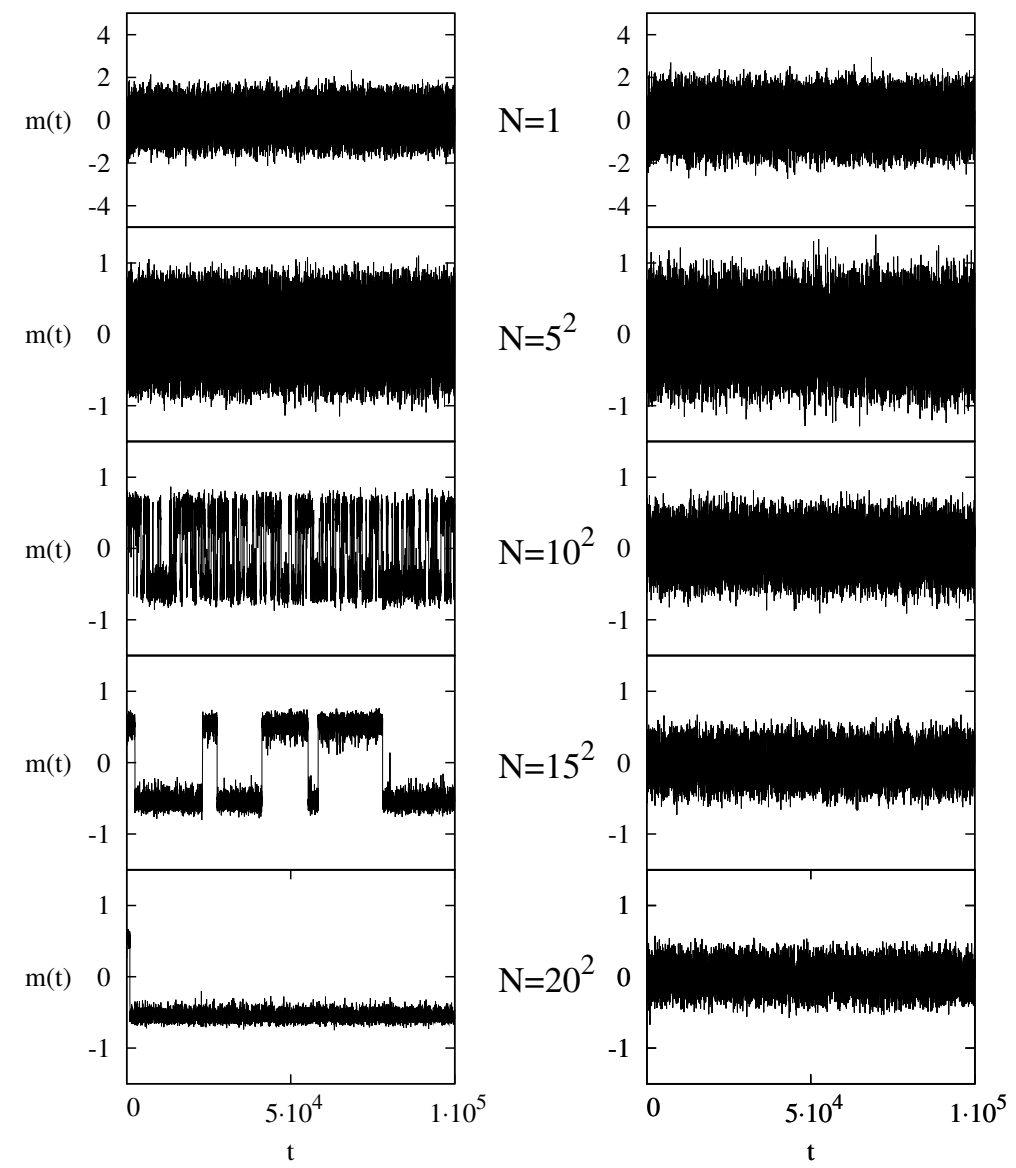

FIGURE 1. Time traces of the magnetization $m(t)=N^{-1} \sum_{i=1}^{N} x_{i}(t)$ for the Ginzburg-Landau model in a 2- $d$ regular network with nearest-neighbors coupling. The right column corresponds to $D=4$ (disordered state), and the left column to $D=1.5$ (ordered stated). In both cases it is $\mu=0.5$ and the coupling constant is $C=20$. Note that the uncoupled system, $N=1$ is always disordered as, in both cases, it has the maximum of the probability distribution located at $x=0$. Note also that the width of the distributions decrease with $N$ and tend to delta-functions in the limit $N \rightarrow \infty$.

It is remarkable, and counterintuitive, that a globally ordered situation arises as a result of an increase of the noise intensity. As it can be seen in Fig.2, the bifurcation is truly symmetry-breaking only for $N \rightarrow \infty$. If noise is increased even further, then a new bifurcation to the disordered state is obtained. However, as explained in detail in $[15,16]$ the explanation of this counterintuitive behavior has to do with the short-time dynamical instability of $x_{i}$ rather than with the long-time steady distribution. We refer the interested reader to those papers and the excellent review in the book [17] for further details on this topic.

Let us now analyze this model using the results of the previous section with $q(x)=-x\left(1+x^{2}\right)^{2}$ and $g(x)=1+x^{2}$. The change of variables in this case is $z=\int^{x} \frac{d x^{\prime}}{1+x^{\prime 2}}=\arctan (x)$ or $x=\tan (z)$. A one-to-one transformation is obtained if 

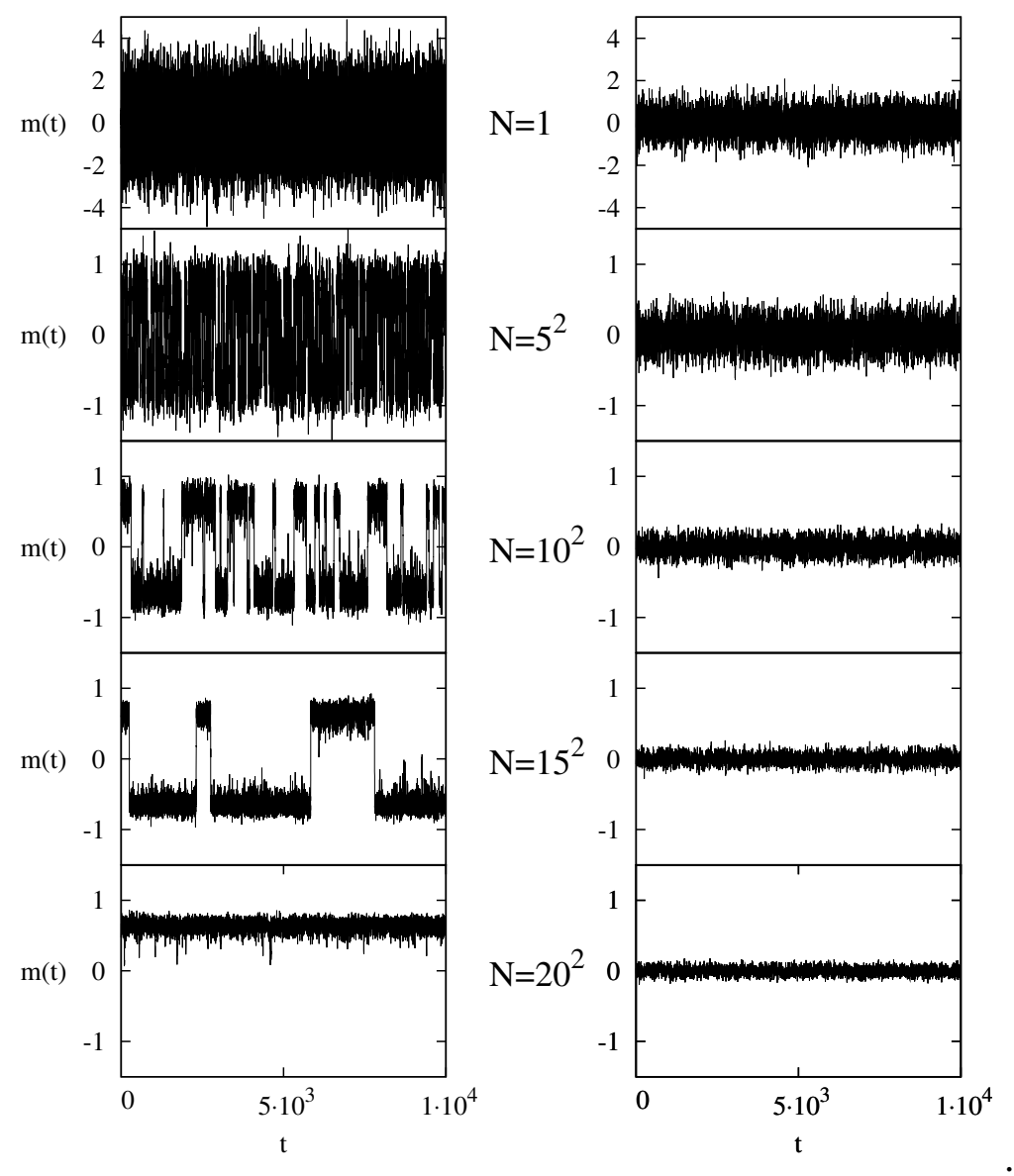

FIGURE 2. Time traces of the magnetization $m(t)=N^{-1} \sum_{i=1}^{N} x_{i}(t)$ for the canonical model displaying a noise-induced phase transition, Eq. (27) in a 2- $d$ regular network with nearest-neighbors coupling. The right column corresponds to $D=0.8$ (disorder state), and the left column to $D=4$ (order induced by noise). The coupling constant is $C=20$ in both cases. As in the previous figure, note that the uncoupled system, $N=1$ is always disordered as, in both cases, it has the maximum of the probability distribution located at $x=0$. Note also that the width of the distributions decrease with $N$ and tend to delta-functions in the limit $N \rightarrow \infty$. Here and in Fig.1, the trajectories have been generated by a stochastic version of the Runge-Kutta algorithm, known as the Heun method [3] and using an efficient generator of Gaussian random numbers [18]

we limit $z \in(-\pi / 2, \pi / 2)$. The Langevin equation for the $z$ variable is

$$
\frac{d z}{d t}=-\frac{\sin (z)}{\cos (z)^{3}}+\sqrt{2 D} \xi(t),
$$

with a potential $V(z)=\frac{1}{2 \cos (z)^{2}}$. The potential is monostable for $z \in(-\pi / 2, \pi / 2)$. The effective potential for the $x$ variable is:

$$
V_{\mathrm{eff}}(x)=\frac{x^{2}}{2}+D \log \left(1+x^{2}\right)
$$


which, again, is always monostable. Therefore, in this case the change of variables does not induce any bistability.

In summary, we have revisited the concept of noise-induced transitions, defined as shifts in the maxima of the steady state probability distribution. They can not be considered "bona fide" bifurcations in the standard sense as (i) they can disappear through a one-to-one change of variables and (ii) there is no true symmetry breaking as all states can be visited independently of the initial condition. A noise-induced phase transition, on the other hand, can appear in the global variable of a coupled system. In the thermodynamic limit it displays symmetry breaking and lack of ergodicity. There are bifurcations from disorder to order when increasing the noise intensity (as in the Ginzburg-Landau model) but, more remarkably, there are cases in which an ordered state can appear as a result of an increase of the noise intensity. Generally, the transition is reentrant, in the sense that a large noise recovers the ordered state, but it is possible to find other situations in which reentrance does not occur [19].

\section{ACKNOWLEDGMENTS}

I thank N. Komin for help in plotting the figures. I acknowledge financial support by the MEC (Spain) and FEDER (EU) through project FIS2007-60327 (FISICOS).

\section{REFERENCES}

1. S. H. Strogatz, Nonlinear dynamics and chaos, Addison-Wesley, Reading, Mass., 1994, second edn.

2. A. Scott, editor, Encyclopedia of Nonlinear Science, Routledge, 2005.

3. M. S. Miguel, and R. Toral, "Stochastic effects in physical systems," in Instabilities and nonequilibrium structures VI, edited by J. M. E. Tirapegui, and R. Tiemann, Kluwer academic publishers, 2000, pp. 35-120.

4. H. Risken, The Fokker-Planck equation, Springer-Verlag, Berlin, 1989, 2nd edn.

5. H. Kramers, Physica (Ultrecht) 7, 284 (1940).

6. N. van Kampen, Stochastic Processes in Physics and Chemistry, North-Holland, Amsterdam, 1981, 1 st edn.

7. C. Gardiner, Handbook of Stochastic Methods for Physics, Chemistry and the Natural Sciences., Springer-Verlag, Berlin, 1983, first edn.

8. W. Horsthemke, and R. Lefever, Noise-Induced Transitions: Theory and Applications in Physics, Chemistry, and Biology, Springer, 1984.

9. M. Hongler, Helv. Phys.Acta 52, 280 (1979).

10. D. J. Amit, and V. M. Mayor, Field Theory, the Renormalization Group and Critical Phenomena, World Scientific Publishing Co.Pte. Ltd., 2005, 3rd edn.

11. R. Albert, and A. Barabasi, Rev. Mod. Phys. 74, 47 (2002).

12. H. Stanley, Introduction to phase transitions and critical phenomena, Oxford university press, 1971.

13. R.Toral, and A. Chakrabarti, Phys. Rev. B 42, 2445 (1990).

14. C. Van den Broeck, J. Parrondo, J. Armero, and A. Hernández-Machado, Phys. Rev. E 49, 2639-2643 (1994).

15. C. van den Broeck, J.M.R. Parrondo, and R. Toral, Phys. Rev. Lett. 73, 3395 (1994).

16. C. van den Broeck, J.M.R. Parrondo, R. Toral, and K. Kawai, Phys. Rev. E 55, 4084 (1997).

17. J. García-Ojalvo, and J. M. Sancho, Noise in Spatially Extended Systems, Springer-Verlag, New York, 1999.

18. R. Toral, and A. Chakrabarti, Computer Physics Communications 74, 327 (1993).

19. M. Ibañes, J. Garcia-Ojalvo, R. Toral, and J.M. Sancho, Phys. Rev. Lett. 87, 20601 (2001). 